\title{
Bottom-Up Fabrication of a Metal-Supported Oxo-Metal Porphyrin
}

D. A. Duncan ${ }^{1,2}$, P. S. Deimel ${ }^{1}$, A. Wiengarten ${ }^{1}$, M. Paszkiewicz ${ }^{1}$, P. Casado Aguilar ${ }^{1 \neq}$, R. G. Acres ${ }^{3 \uparrow,}$ F. Klappenberger ${ }^{1}$, W. Auwärter ${ }^{1}$, A. P. Seitsonen ${ }^{4,5}$, J. V. Barth ${ }^{1}$ and F. Allegretti ${ }^{1}$

${ }^{1}$ Physics Department E20, Technical University of Munich, D-85748 Garching, Germany

2 Diamond Light Source, Harwell Science and Innovation Campus, Didcot, OX11 0QX, United Kingdom

${ }^{3}$ Elettra-Sincrotrone Trieste S.C.p.A., S.S. 14-km 163.5, 34149 Basovizza, Trieste, Italy

${ }^{4}$ Département de Chimie, École Normale Supérieure, 24 rue Lhomond, F-75005 Paris, France

${ }^{5}$ Université de recherche Paris-Sciences-et-Lettres, Sorbonne Université, Centre National de la Recherche Scientifique, F-75005 Paris, France

‡Present address: Instituto Madrileño de Estudios Avanzados en Nanociencia, Cantoblanco 28049, Madrid, Spain

"Present address: Australian Synchrotron, 800 Blackburn Road, Clayton, Victoria, 3168, Australia

Corresponding authors:

francesco.allegretti@ph.tum.de

david.duncan@diamond.ac.uk

\section{Supporting Information (SI)}


Table S1. Fitted binding energy (BE) and full width at half maximum (FWHM) values for the SXPS (N $1 s$ and $\mathrm{O} 1 s)$ and XPS (Ti 2p) spectra shown in Figure $2(\mathrm{~N} 1 s)$, Figure 5 (Ti 2p) and Figure $6(\mathrm{O} 1 s)$ in the main article.

\begin{tabular}{|c|c|c|c|c|c|c|c|}
\hline & \multirow[b]{2}{*}{ system } & \multicolumn{2}{|c|}{$-N=$} & \multicolumn{2}{|c|}{$-\mathrm{NH}-$} & \multicolumn{2}{|c|}{$\mathrm{N}-\mathrm{Ti}$} \\
\hline & & $\mathrm{BE}(\mathrm{eV})$ & FWHM (eV) & $\mathrm{BE}(\mathrm{eV})$ & FWHM (eV) & $\mathrm{BE}(\mathrm{eV})$ & FWHM $(\mathrm{eV})$ \\
\hline \multirow{3}{*}{$\mathrm{N} 1 \mathrm{~s}$} & $2 \mathrm{H}-\mathrm{TPP}$ & 398.1 & 1.01 & 400.0 & 1.19 & - & - \\
\hline & Ti-TPP & 398.1 & 0.99 & 400.1 & 1.21 & 398.6 & 0.99 \\
\hline & TiO-TPP & 398.1 & 1.03 & 400.0 & 1.21 & 398.6 & 1.10 \\
\hline
\end{tabular}

$\mathrm{Ti}(\mathrm{II})$

$\mathrm{Ti}($ II) satellite

Ti (IV)

\begin{tabular}{|c|c|c|c|c|c|c|c|}
\hline & system & $\mathrm{BE}(\mathrm{eV})$ & FWHM (eV) & $\mathrm{BE}(\mathrm{eV})$ & FWHM (eV) & $\mathrm{BE}(\mathrm{eV})$ & FWHM (eV) \\
\hline Ti $2 p_{3 / 2}$ & Ti-TPP & $455.8(461.7)$ & $2.90(2.66)$ & $458.9(464.8)$ & $2.90(2.66)$ & - & - \\
\hline (Ti $\left.2 p_{1 / 2}\right)$ & TiO-TPP & 455.7 (461.6) & $2.13(2.68)$ & - & - & $457.8(463.7)$ & $2.13(2.68)$ \\
\hline
\end{tabular}

beam stable species beam sensitive species

system energy (eV) FWHM (eV) energy (eV) FWHM (eV)

\begin{tabular}{cccccc}
\hline \hline O 1s & TiO-TPP & 529.6 & 1.38 & 531.8 & 1.60 \\
\hline
\end{tabular}
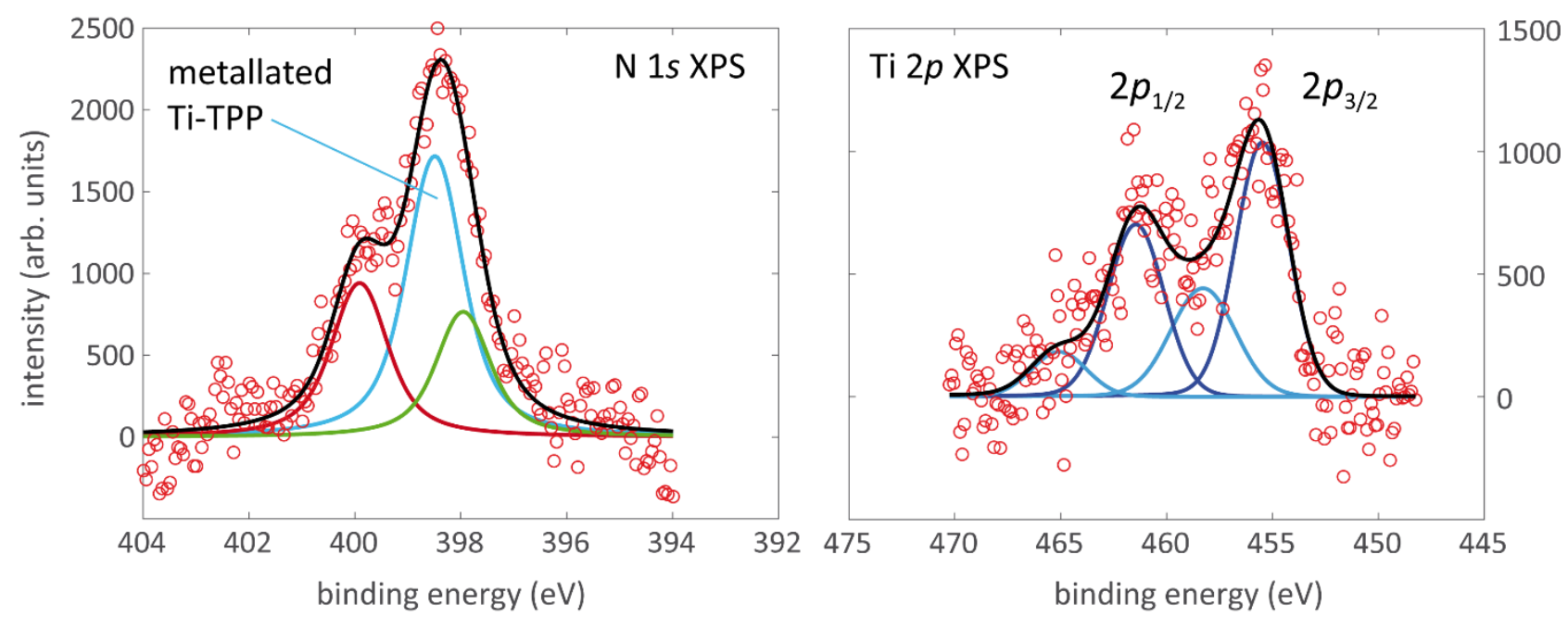

Figure S1: Fitted $\mathrm{N} 1 s$ and Ti $2 p \mathrm{XP}$ spectra with $\mathrm{Al} K \alpha$ radiation acquired in the same electron emission geometry for a partially metalated layer of Ti-TPP and 2H-TPP species. Comparing the peak area of the $\mathrm{N} 1 s$ metalated component and the total area of the Ti $2 p$ XP line and correcting for the relative photoionization cross sections, a ratio of $4.1 \pm 0.4: 1$ is found, confirming that all $\mathrm{Ti}$ on the surface has been incorporated into the TPP molecules. 

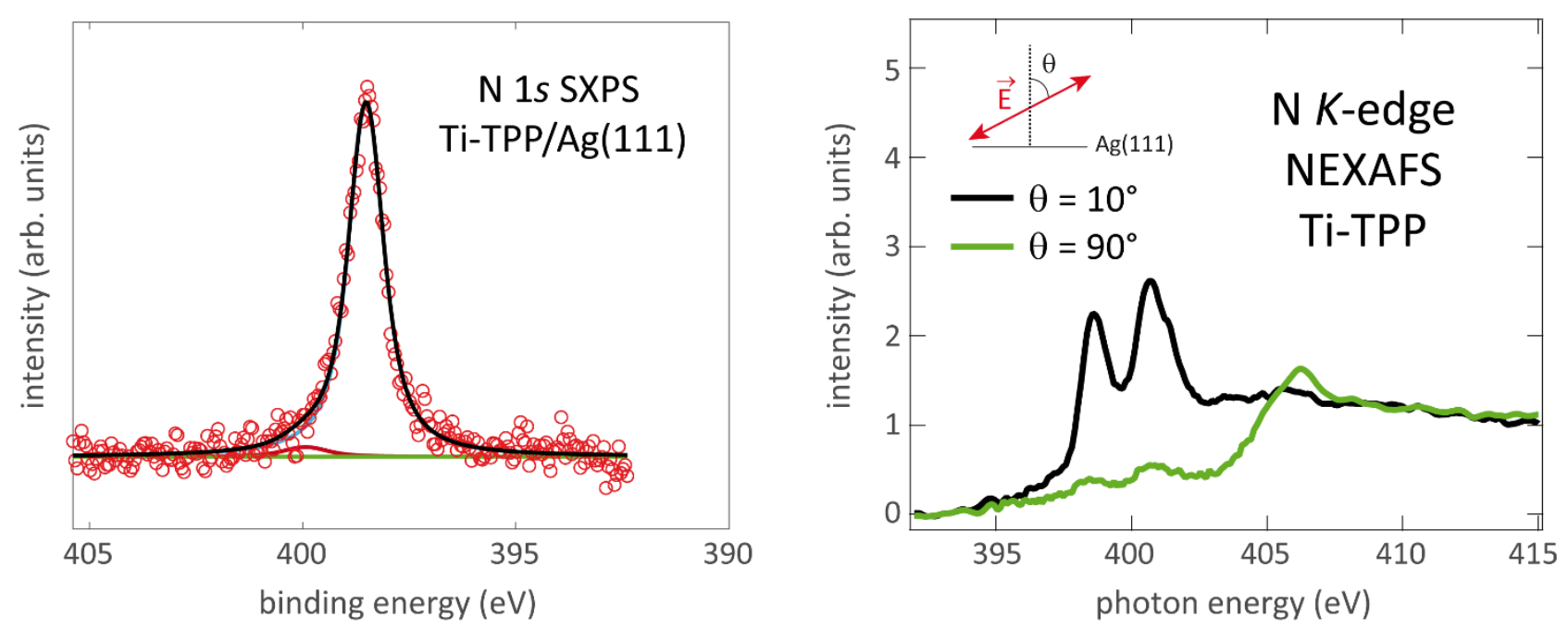

Figure S2: (left) Fitted N 1s SXP spectrum acquired with synchrotron radiation at a photon energy of $550 \mathrm{eV}$ for a fully metalated layer of Ti-TPP on Ag(111); (right) $\mathrm{N} K$-edge NEXAFS measurements for the same Ti-TPP monolayer. The angle $\theta$ in the NEXAFS data defines the light polarization direction with respect to the surface normal (inset).
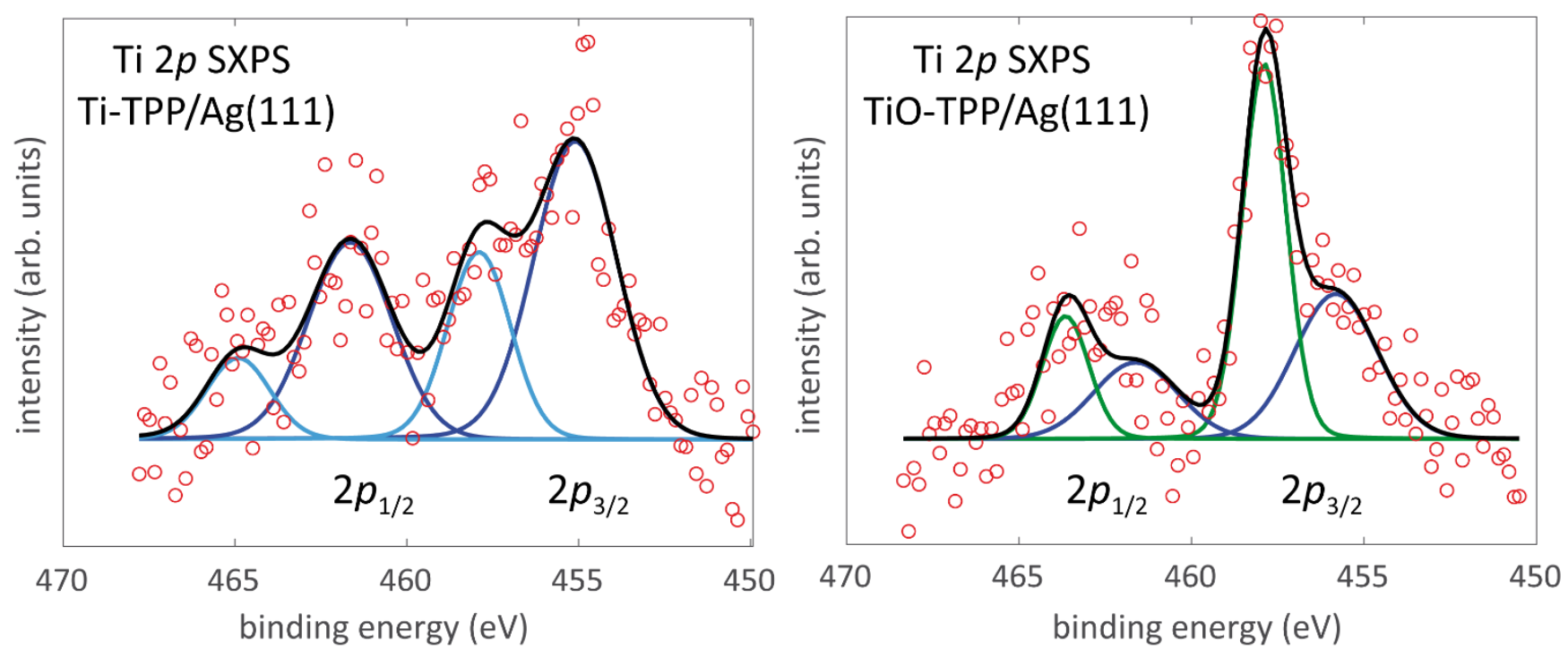

Figure S3: Fitted Ti $2 p$ SXP spectra acquired with synchrotron radiation at a photon energy of $680 \mathrm{eV}$ for the layers of Figures $2 c, 2 d, 5 b$ (left panel) and Figure 2e, 2f, $5 d$ (right panel), respectively. The sharpening of the $2 p_{3 / 2}$ and $2 p_{1 / 2}$ photoemission lines is observed. For TiO-TPP an oxygen exposure of $200 \mathrm{~L}$ (at room temperature) was used. 


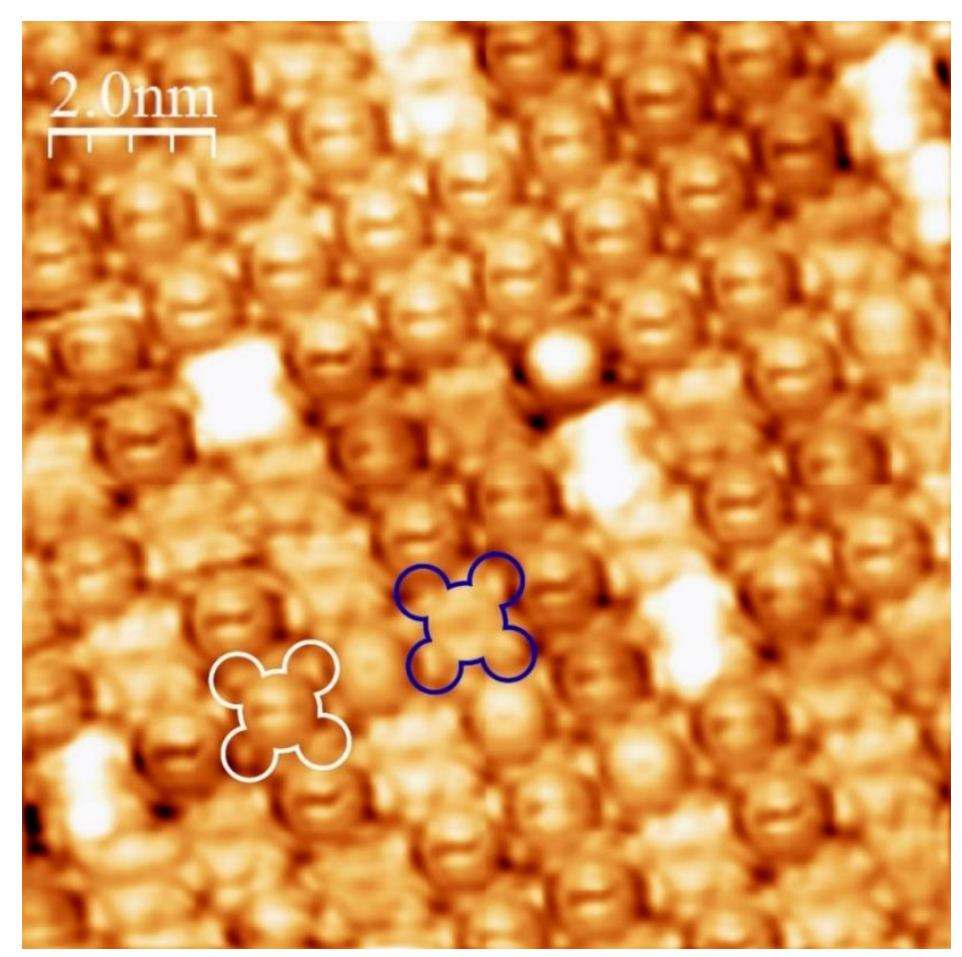

Figure S4: STM image of TiO-TPP on Ag(111), acquired utilizing the "special tip conditions" described in the main article. The image displays a somewhat enhanced contrast between (white) TiO-TPP and (dark blue) $2 \mathrm{H}$-TPP. Both molecular species are highlighted by the overlaid contours. Bias: $-0.6 \mathrm{~V}$; Current: $0.11 \mathrm{nA}$.

\section{DFT: occupied density of states}

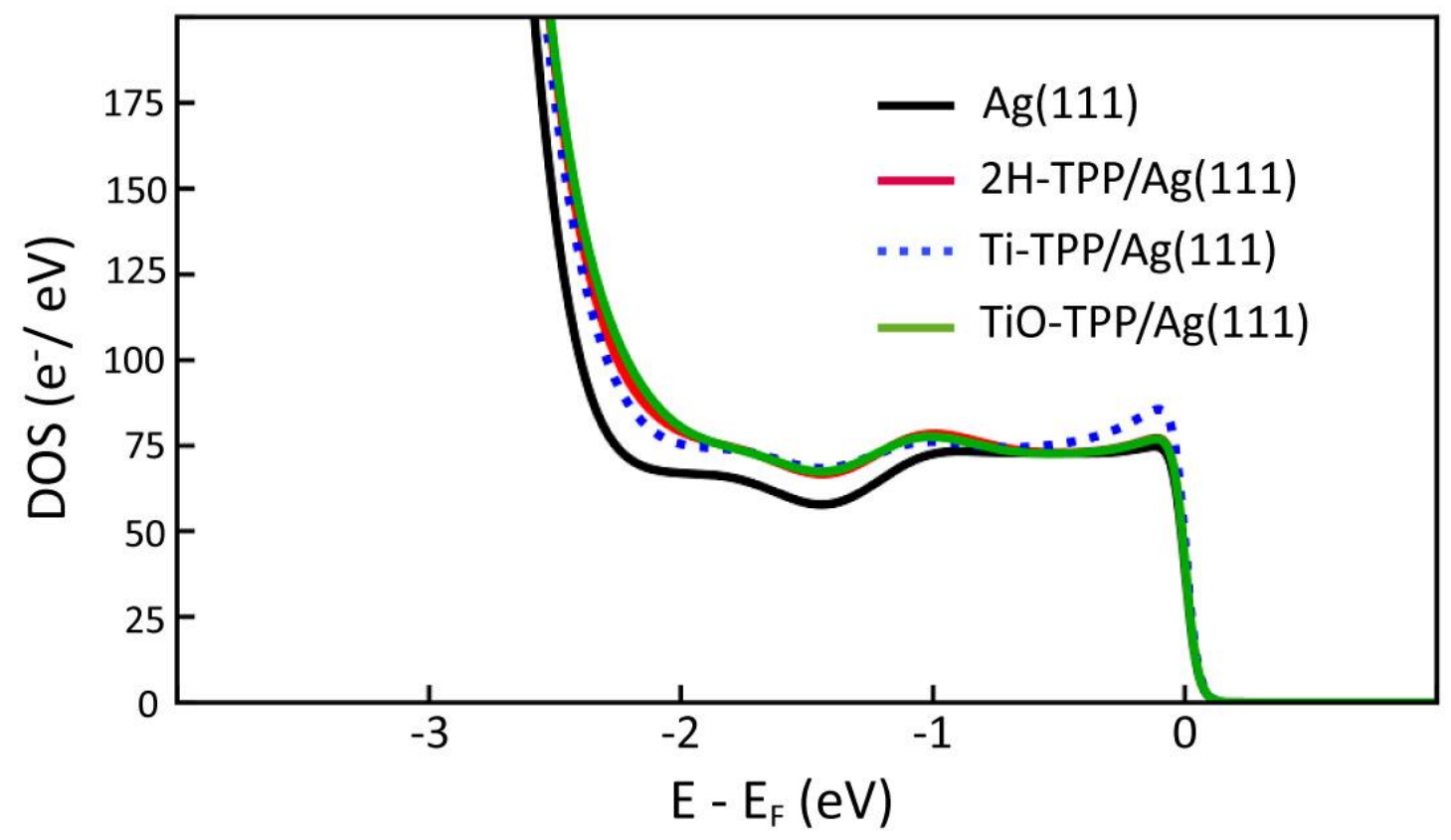

Figure S5: Calculated density of states (DOS) from density functional theory (DFT) for the $\operatorname{Ag}(111)$ surface and the different molecules adsorbed on it. The energy scale is referred to the Fermi level. 


\section{TiO-TPP monolayer / $\mathrm{Ag}(\mathbf{1 1 1})$}

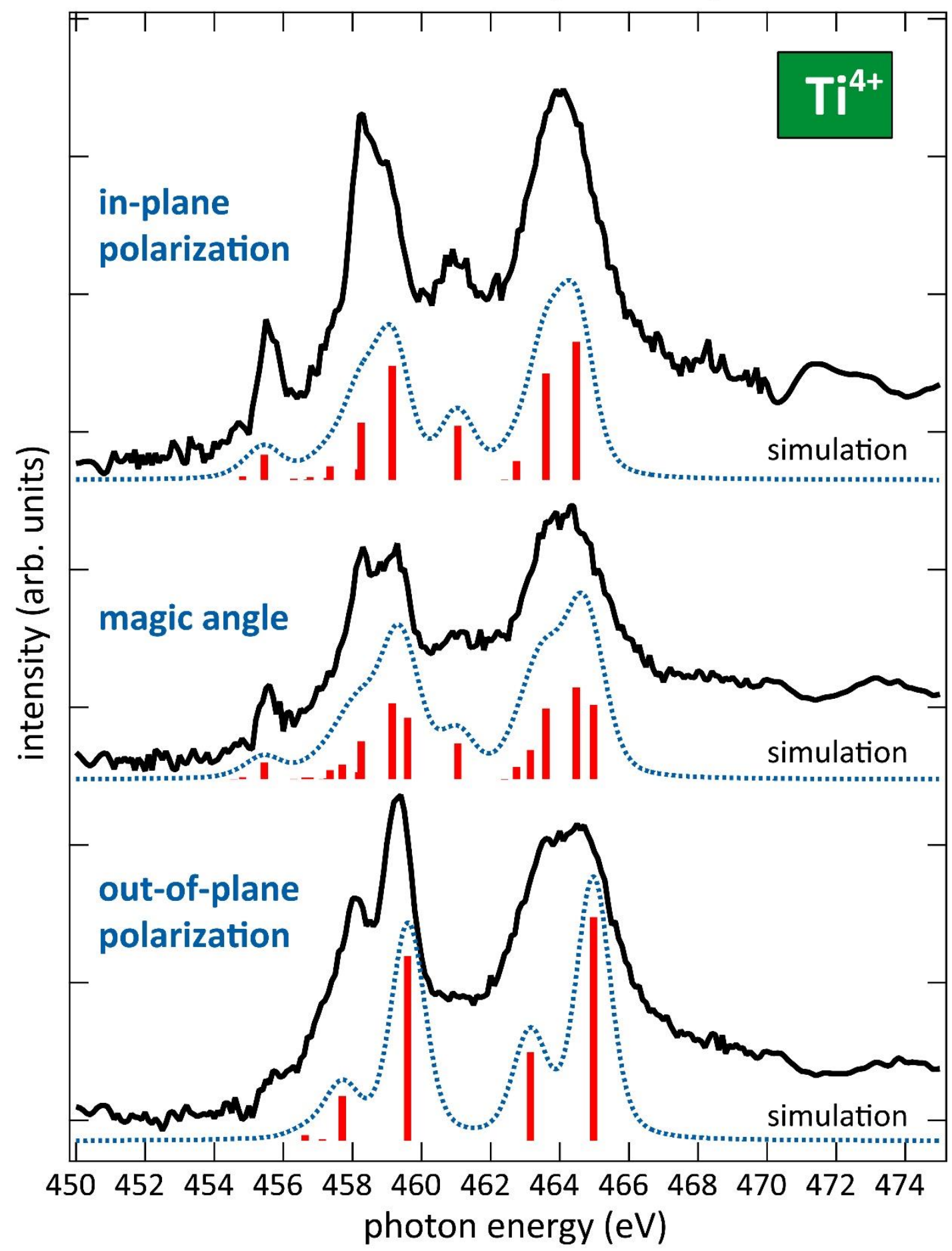

Figure S6: Comparison of experimental (black) and simulated (blue, dotted) Ti L-edge NEXAFS spectra for TiO-TPP/Ag(111) in different polarization geometries. The simulated spectra were calculated using the CTM4XAS program. Top panel: polarization in the plane of the molecules (experiment: $\theta=90^{\circ}$ from the surface normal); middle panel: magic angle (experiment: $\theta=52^{\circ}$ ); bottom panel: polarization perpendicular to the plane of the molecules (experiment: $\theta=10^{\circ}$ ). The simulated spectra were multiplied by a common factor for better comparison. Sticks (in red) correspond to the $d$-orbital split levels as calculated by the CTM4XAS program. Deviations from the planarity and the exact orientation of the molecules on $\mathrm{Ag}(111)$ are not taken into account in the simulations, which might be the reason for the intensity dependence on the polarization not being accurately reproduced. 


\section{Ti-TPP monolayer / $\mathrm{Ag}(\mathbf{1 1 1})$}

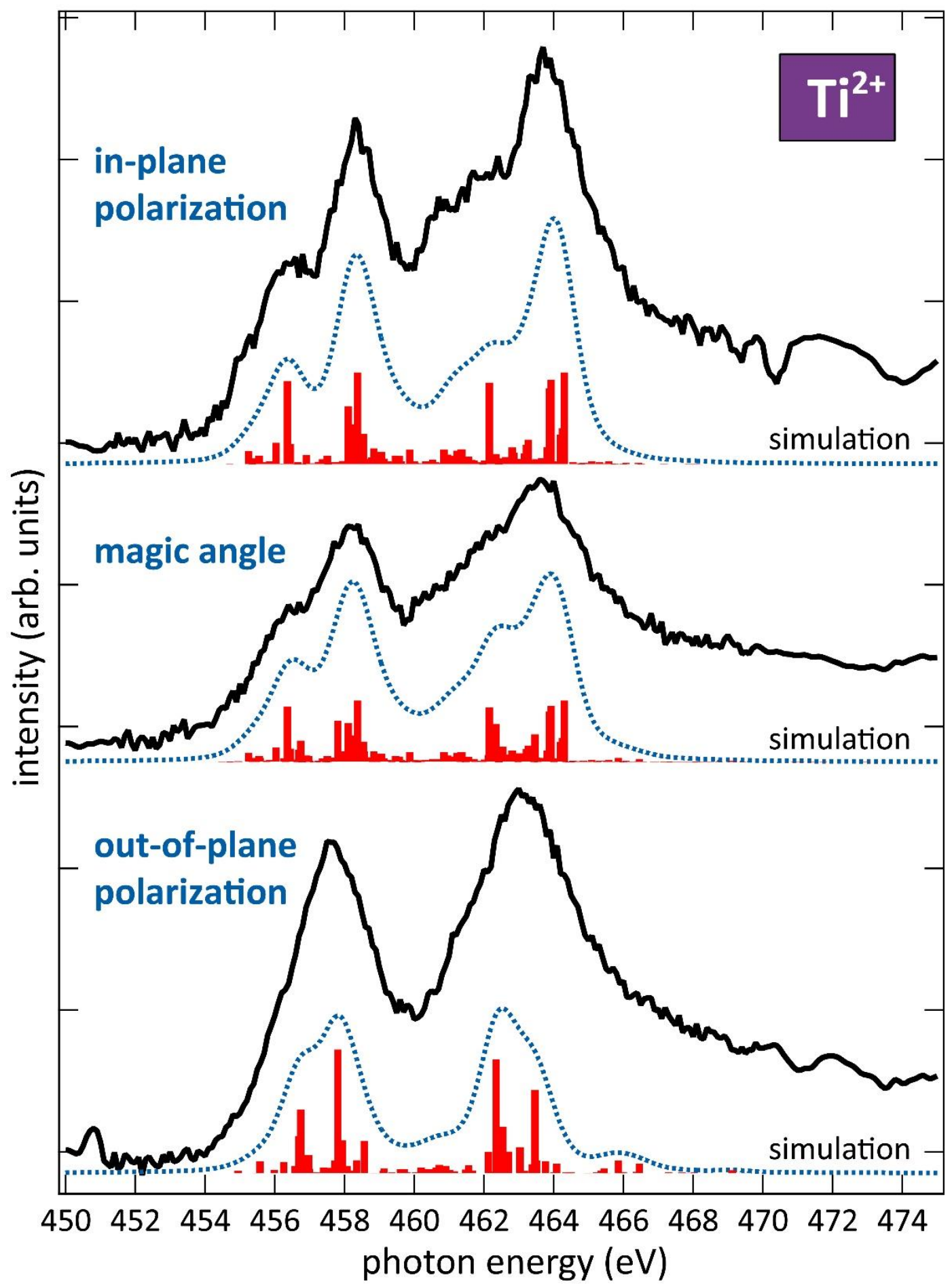

Figure S7: Comparison of experimental (black) and simulated (blue, dotted) Ti L-edge NEXAFS spectra for Ti-TPP/Ag(111) in different polarization geometries. The simulated spectra were calculated using the CTM4XAS program. Top panel: polarization in the plane of the molecules (experiment: $\theta=90^{\circ}$ from the surface normal); middle panel: magic angle (experiment: $\theta=52^{\circ}$ ); bottom panel: polarization perpendicular to the plane of the molecules (experiment: $\theta=10^{\circ}$ ). The simulated spectra were multiplied by a common factor for better comparison. Sticks (in red) correspond to the $d$-orbital split levels as calculated by the CTM4XAS program. A rigid energy shift of $+2.2 \mathrm{eV}$ was applied to the simulated data (see main article). Deviations from the planarity and the exact orientation of the molecules on $\mathrm{Ag}(111)$ are not taken into account in the simulations, which might be the reason for the intensity dependence on the polarization not being accurately reproduced. 


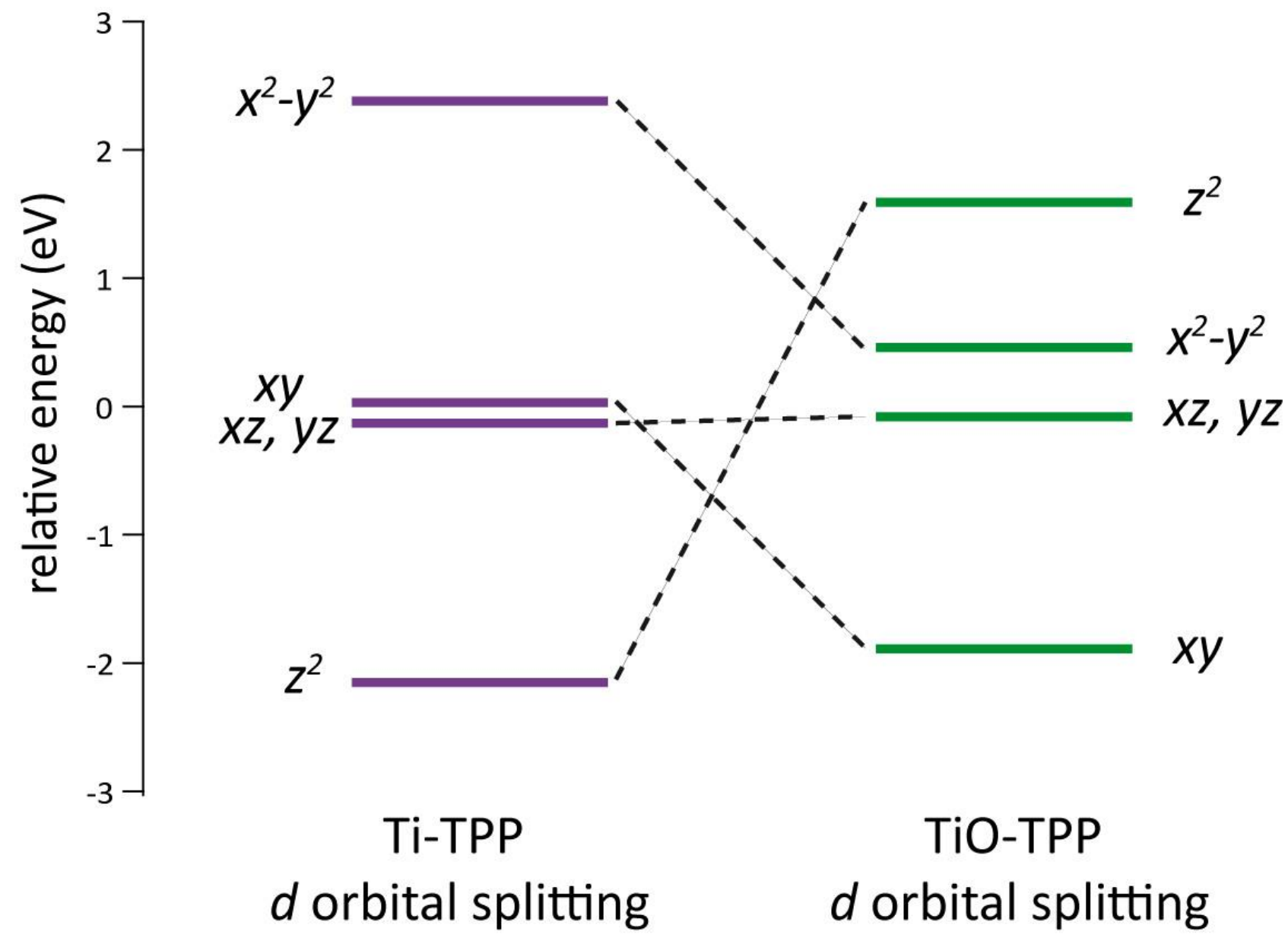

Figure S8: One-electron energy levels for the $3 d$ orbitals of the adsorbed Ti-TPP (left) and TiO-TPP (right), determined from the CTM4XAS simulations. The changes upon oxygen coordination are highlighted through the black dashed lines. The relative energies of the levels in the manifold were calculated using the following equations (cf. [1] and references therein) with the crystal-field parameters provided by the L-edge NEXAFS simulations:

(1) $b_{1 g}\left(d_{x^{2}-y^{2}}\right)=+6 D q+2 D s-1 D t$

(2) $a_{1 g}\left(d_{z^{2}}\right)=+6 D q-2 D s-6 D t$

(3) $b_{2 g}\left(d_{x y}\right)=-4 D q+2 D s-1 D t$

(4) $e_{g}\left(d_{x z, y z}\right)=-4 D q-1 D s+4 D t$ 

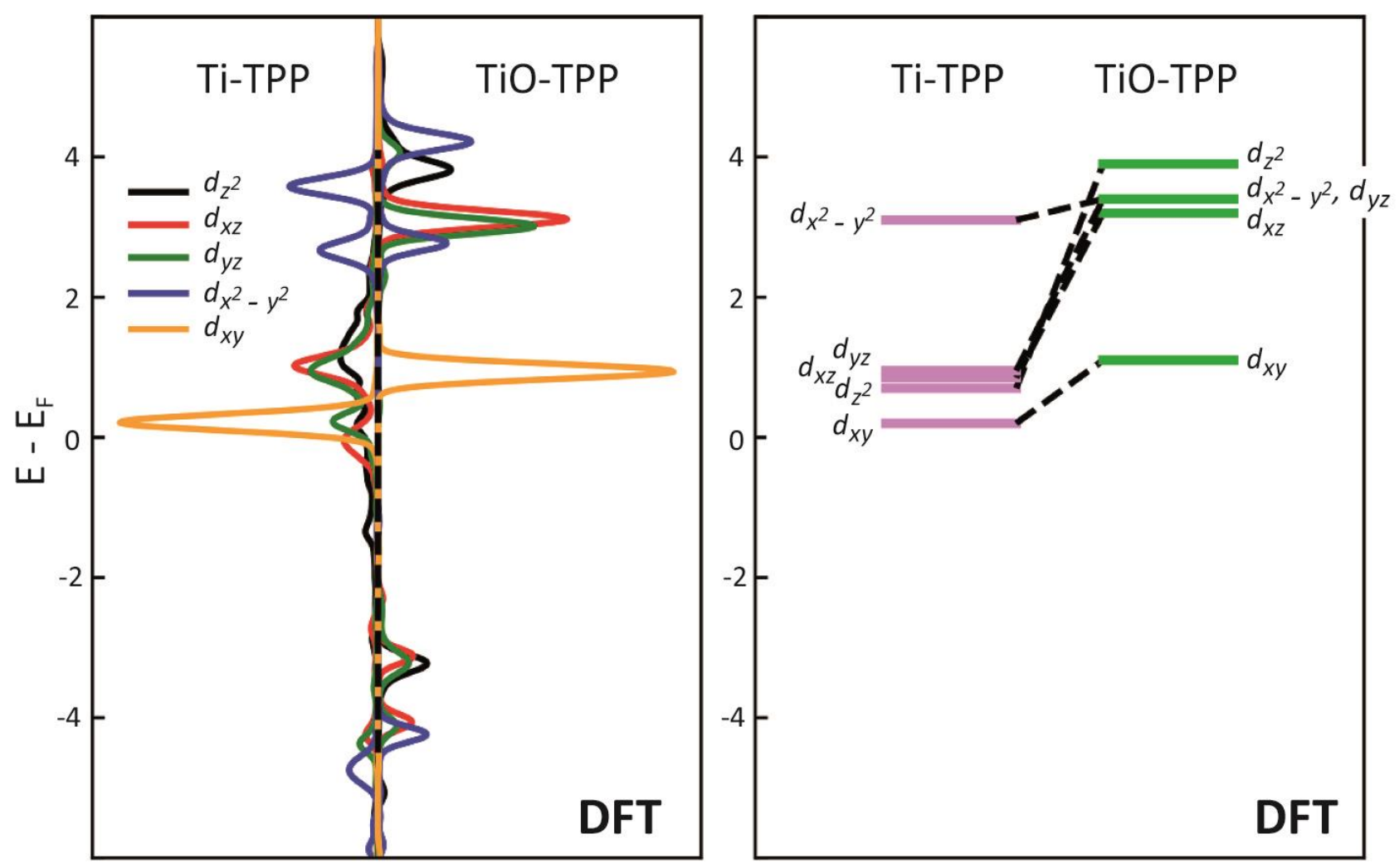

Figure 59: (left panel) Projected density of states (PDOS) on the transition metal $d$ orbitals calculated by DFT. (right panel) Schematization of the energy level ordering to enable visual comparison with Figure S8. Here, for the split and broadened states of given symmetry, only the center of gravity of the unoccupied states was considered. While there is qualitatively good agreement of this (simplified) energy diagram with the one-electron picture of Figure $\mathrm{S} 8$ for TiO-TPP, more pronounced differences are observed for Ti-TPP. This is presumably related to the stronger interaction of the metal center with the Ag surface and the different molecular conformation predicted by DFT (see text), which are not accounted for in the L-edge NEXAFS simulations.

\section{References}

[1] Pickup, D. F.; Zegkinoglou, I.; Ballesteros, B.; Ganivet, C. R.; García-Lastra, J. M.; Cook, P. L.; Johnson, P. S.; Rogero, C.; de Groot, F.; Rubio, A., et al., Influence of Axial and Peripheral Ligands on the Electronic Structure of Titanium Phthalocyanines. J. Phys. Chem. C 2013, 117, 4410-4420. 